\title{
Energy Detection based Blind Synchronization for Pulse Shape Modulated IR-UWB Systems
}

\author{
R. Akbar ${ }^{1-2}$, E. Radoi ${ }^{1-2}$, and S. Azou ${ }^{1-2}$ \\ ${ }^{1}$ Université Européenne de Bretagne, France \\ ${ }^{2}$ Université de Brest; CNRS, UMR 3192 Lab-STICC, ISSTB, \\ 6 avenue Victor Le Gorgeu, CS 93837, 29238 Brest cedex 3, France \\ \{rizwan.akbar, emanuel.radoi\}@univ-brest.fr \\ http://www.lab-sticc.fr
}

\begin{abstract}
Synchronization is a key performance-limiting factor in any communication system and a challenging task to accomplish. In this paper, an energy detection based non dataaided (NDA) algorithm for orthogonal pulse shape modulated (PSM) impulse radio ultra wideband (IR-UWB) system is proposed. Relying on unique signal structure, simple overlap-add operation followed by energy detection enables synchronization. The algorithm remains functional under practical scenarios i.e. in the presence of inter-frame and inter-symbol interference (IFI \& ISI) and with M-ary modulation. Simulation results are provided to demonstrate the performance of proposed algorithm.

Index Terms-Blind synchronization, IR-UWB, Orthogonal pulse shape modulation, Energy detection.
\end{abstract}

\section{INTRODUCTION}

Ultra wideband (UWB) radio has gained considerable attention since Federal Communication Commission (FCC) ruling in 2002, approving it for data communications as well as for radar applications. Impulse based UWB (IR-UWB) is characterized by transmission of ultra short UWB pulses in a discontinuous way, occupying a staggering band of $7.5 \mathrm{GHz}$ with extremely low power density of $-41.25 \mathrm{dBm} / \mathrm{MHz}$. It is envisioned as a promising candidate for short range indoor wireless communications, on account of several captivating features such as low-complexity low-cost transceiver, ability to overlay existing narrowband systems, ample multipath diversity, high data-rate and precise ranging at centimeter level [1].

Synchronization is crucial to benefit from aforementioned features of UWB and a pronounced BER performance degradation is observed in the presence of imperfect timing [2], [3]. Though synchronization is a big challenge in any communication system, its difficulty is magnified in IR-UWB due to impulsive and low power nature of UWB pulses, unknown channel with dense multipath and inevitable IFI \& ISI when aiming for high data-rate [4].

Recently, the possibility of generating multiple mutually orthogonal and spectrally efficient pulses with same widths have motivated the use of orthogonal modulation schemes such as pulse shape modulation (PSM) for IR-UWB [5], [6] systems, instead of conventional pulse amplitude modulation

This research work has been supported by Brittany Region and Institut Supérieur de Sciences et Technologies de Brest (ISSTB), France.
(PAM) and pulse position modulation (PPM). Although orthogonal signaling performs $3 \mathrm{~dB}$ poorer than PAM in binary modulation [7], it outclasses latter for higher order modulations i.e. $M \geq 4$, which is essential to design high data-rate systems [7]. This feature along with its possible robustness against multi-access interference and ISI makes PSM the focus of this paper.

Several non data-aided (NDA) algorithms have been developed to address the issue of synchronization; however most of them are for binary PAM and are not readily applicable to orthogonal modulations. Generally, these algorithms can be categorized in (1) Correlation (2) Energy Detection and (3) Code Matching based methods. In correlation based methods, synchronization is achieved by peak-picking the cross correlation samples of adjacent received signal segments [8], [9]. The energy detection based methods rely on judiciously designed signal structure, which upon first order averaging will cancel out whole signal except the particular portion later used by energy detector to accomplish synchronization [10], [11]. The third class of methods employs bipolar codes with perfect autocorrelation and cross correlation properties (ACP). The correlation between received signal and code followed by aggregation will achieve synchronization [4], [12].

Unfortunately, the criterion of peak-picking as well as cancellation with averaging will not remain applicable in orthogonal PSM. It is worth mentioning that some of above algorithms will work if data-aided (DA) approach is considered, however training sequence will reduce bandwidth efficiency which is not appealing for high speed networks. Thus our objective here is to develop NDA approach for synchronization in orthogonal PSM. The rest of this paper is laid out as follows. Section II briefs out signal format and preliminaries. Synchronization algorithm is described in section III. Simulations are presented in sections IV while conclusions are summarized in section V.

\section{SYSTEM MODEL}

\section{A. PSM Transmission Model}

For a typical IR-UWB transmission equipped with timehopping (TH) codes, the transmitted signal employing orthogonal PSM modulation is given by

$$
s(t)=\sum_{i} g_{T, d(i)}\left(t-i T_{s}\right)
$$


where

$$
g_{T, d(i)}(t)=\sum_{j=0}^{N_{f}-1} \psi_{d(i)}\left(t-j T_{f}-c_{j} T_{c}\right)
$$

is symbol-long transmitted waveform. Each informationbearing symbol $d(i) \in\{0,1, \ldots, M-1\}$ is conveyed using one of ultra-narrow orthogonal UWB pulse in the set $S=$ $\left\{\psi_{0}(t), \psi_{1}(t), \ldots, \psi_{M-1}(t)\right\}$, each of duration $T_{\psi}$ and satisfying $\int_{0}^{T_{\psi}} \psi_{i}(t) \psi_{j}(t)=\delta(i-j)$ for $(i, j)=0,1, \ldots, M-1$. Due to severe limitations imposed by FCC on transmission power, UWB pulses are repeated over $N_{f}$ frames with one pulse per frame to represent data symbol $d(i)$, thereby increasing effective SNR per symbol. The symbol duration is thus $T_{s}=N_{f} T_{f}$ where $T_{f}$ is frame duration. Spectrum smoothing and multi-access are established by time shifting UWB pulses at multiples of chip duration $T_{c}$ using user-specific pseudorandom TH codes $c_{j} \in\left[0, N_{h}\right)$, with $N_{h} \leqslant N_{c}$ where $N_{c}$ are number of chips per frame satisfying $T_{f}=N_{c} T_{c}$.

\section{B. Modified PSM Transmission Model}

To ease out synchronization, the conventional transmission model of PSM in (2) is modified as following

$$
g_{T, d(i)}(t)=\psi_{0}\left(t-c_{0} T_{c}\right)+\gamma_{i} \sum_{j=1}^{N_{f}-1} \psi_{d(i)}\left(t-j T_{f}-c_{j} T_{c}\right)
$$

where

$$
\gamma_{i}=\sum_{m} \alpha_{i}^{m} \beta_{i}^{m}, \quad m=0,1, \ldots, M-1
$$

with

$$
\begin{aligned}
\beta_{i}^{m} & =\beta_{i-1}^{m}\left[1-2 \alpha_{i}^{m}\right] \\
\alpha_{i}^{m} & =\left[\left\lceil\frac{|d(i)-m|+M}{M}\right\rceil\right]_{\bmod 2}
\end{aligned}
$$

where $\alpha_{i}^{m} \in\{0,1\}$ and $\left\{\beta_{i}^{m}, \gamma_{i}\right\}= \pm 1$ ( $\lceil$.$\rceil stands for integer$ ceil operation). From (3), it is clear that two changes have been made. First, the starting frame of each symbol is reserved and can be regarded as information-free pulse. Without loss of generality, we set $c_{0}=0$ hereafter. Secondly, pulses with alternate phase are used to represent a particular symbol i.e. the data symbol $d(i)$ is transmitted using $\psi_{d(i)}(t)$ and $-\psi_{d(i)}(t)$ alternatively. The graphical explanation of these changes can be observed in Fig. 1.

\section{Reception Model}

The transmitted signal propagates through frequency selective multipath channel which can be modelled as tapped-delay line model with real impulse response $h(t)=\sum_{l=0}^{L-1} \lambda_{l} \delta(t-$ $\left.\tau_{l}\right)$, where $\left\{\lambda_{l}, \tau_{l}\right\}_{l=0}^{L-1}$ are channel path gains and delays respectively, satisfying $\tau_{l}<\tau_{l+1}, \forall l$. The UWB channel is typically assumed to be quasi-static i.e. channel taps remain invariant over a block of symbols but may vary from block to block. In order to isolate propagation delay $\tau_{0}$ from channel delays, channel response can be rewritten as $h(t)=\sum_{l=0}^{L-1} \lambda_{l} \delta\left(t-\tau_{l, 0}-\tau_{0}\right)$ where $\tau_{l, 0}=\tau_{l}-\tau_{0}$ is the $d(0)=0|d(1)=2| d(2)=3|d(3)=3| d(4)=0|d(5)=2| d(6)=1|d(7)=1| d(8)=2 \mid$

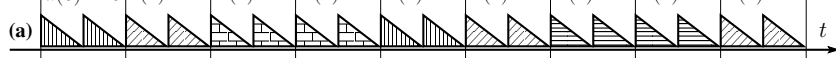

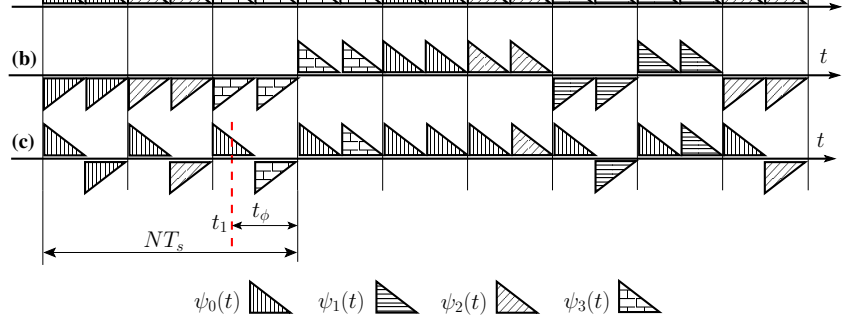

Figure 1. Received Signal in 4-ary Modulation with synchronization parameter $t_{\phi}$ and $N_{f}=2$ (a) Original PSM signal format (b) Alternate signal inversion (c) Information-free first frame, resulting in modified PSM signal format

relative path delay. The received signal is then the convolution of $s(t) * h(t)$ in the presence of additive white Gaussian noise $w(t)$ with double-sided power spectral density $N_{0} / 2$ or equivalently

$$
r(t)=\sum_{i} g_{R, d(i)}\left(t-i T_{s}-\tau_{0}\right)+w(t)
$$

where

$$
g_{R, d(i)}(t)=p_{0}(t)+\gamma_{i} \sum_{j=1}^{N_{f}-1} p_{d(i)}\left(t-j T_{f}-c_{j} T_{c}\right)
$$

is the received aggregate symbol-long waveform with $p_{d(i)}(t)=\sum_{l=0}^{L-1} \lambda_{l} \psi_{d(i)}\left(t-\tau_{l, 0}\right)$ representing channel response to single UWB pulse $\psi_{d(i)}\left(t+\tau_{0}\right)$. Let $T_{p}:=$ $\sup \left\{t \mid p_{d(i)}(t) \neq 0\right\}$ with $T_{p}=T_{\psi}+\tau_{L-1,0}$, then it can readily be seen that $T_{g}=\left(N_{f}-1\right) T_{f}+c_{N_{f}-1} T_{c}+T_{p}$ where $T_{g}:=\sup \left\{t \mid g_{R, d(i)}(t) \neq 0\right\}$.

\section{BLIND SyNCHRONIZATION AlgORITHM}

\section{A. Problem Formulation}

In practical scenarios, receiver is unaware of transmission starting time and channel propagation delay $\tau_{0}$. We assume that receiver initiates synchronization at time $t_{1} \geq \tau_{0}$ and also set $\tau_{0}=0$ as it is only serving as a reference. Denoting $t_{1}$ as an integer multiple of symbol duration and residue i.e. $t_{1}=N T_{s}-t_{\phi}$, the received signal in (6) thus can be rewritten as

$$
\begin{aligned}
x(t) & =r\left(t+t_{1}\right) \\
& =\sum_{i} g_{R, d(i)}\left(t-i T_{s}+t_{1}\right)+w\left(t+t_{1}\right) \\
& =\sum_{i} g_{R, d(i)}\left(t-(i-N) T_{s}-t_{\phi}\right)+w\left(t+N T_{s}-t_{\phi}\right)
\end{aligned}
$$

where $N=\left\lceil t_{1} / T_{s}\right\rceil$ and $t_{\phi} \in\left[0, T_{s}\right)$ is the goal of synchronization algorithm to estimate.

\section{B. Synchronization Algorithm}

Given $x(t)$, a simple energy detection (ED) based algorithm is proposed exploiting the judiciously designed signal format 
of (3). First, we take $T_{s}$-long $K$ segments from received signal $x(t)$, given by

$$
\begin{aligned}
x_{k}(t) & =x\left(t+k T_{s}\right), \quad k \in[0, K-1], t \in\left[0, T_{s}\right) \\
& =\sum_{i} g_{R, d(i)}\left(t-(i-N-k) T_{s}-t_{\phi}\right)+\eta(t)
\end{aligned}
$$

where $\eta(t)=w\left(t+(N+k) T_{s}-t_{\phi}\right)$. Assuming ISI/IFI free scenario (i.e. $T_{g} \leq T_{s}$ and $T_{p} \leq T_{f}$ ), it is easy to observe that each segment $x_{k}(t)$ of size $T_{s}$ will span at most two symbols of $g_{R, d(i)}(t)$. Letting $i=N+k+q$ where $q=0$ or $q=-1$, (9) can be rewritten as

$$
x_{k}(t)=\sum_{q=-1}^{0} g_{R, d(N+k+q)}\left(t-q T_{s}-t_{\phi}\right)+\eta(t)
$$

Next, the mean of observation signal is found using sample mean estimator obtained from $K$ segments as follows

$$
\begin{aligned}
\bar{x}(t) & =\frac{1}{K} \sum_{k=0}^{K-1} x_{k}(t) \\
& =\frac{1}{K} \sum_{k=0}^{K-1} \sum_{q=-1}^{0} g_{R, d(N+k+q)}\left(t-q T_{s}-t_{\phi}\right)+\bar{\eta}(t)
\end{aligned}
$$

where $\bar{\eta}(t)$ is averaged noise. Ignoring noise brevity and substituting (6) in (11), we get

$$
\begin{gathered}
\bar{x}(t)=\sum_{q=-1}^{0} p_{0}\left(t-q T_{s}-t_{\phi}\right)+\frac{1}{K} \sum_{q=-1}^{0} \sum_{k=0}^{K-1} \sum_{j=1}^{N_{f}-1} \gamma_{N+k+q} \\
p_{d(N+k+q)}\left(t-q T_{s}-j T_{f}-c_{j} T_{c}-t_{\phi}\right)
\end{gathered}
$$

From (5), it can be seen that

$$
\alpha_{i}^{m}=\left\{\begin{array}{ll}
1 & \text { if } d(i)=m \\
0 & \text { otherwise }
\end{array}, \beta_{i}^{m}= \begin{cases}-\beta_{i-1}^{m} & \text { if } d(i)=m \\
\beta_{i-1}^{m} & \text { otherwise }\end{cases}\right.
$$

therefore $\alpha_{i}^{m} \beta_{i}^{m}=-\beta_{i-1}^{m}$ for $d(i)=m$, resulting in mean of

$$
\frac{1}{K} \sum_{k=0}^{K-1} \alpha_{k}^{m} \beta_{k}^{m}= \begin{cases}-1 / K & \text { if } p^{m} \text { is odd } \\ 0 & \text { otherwise }\end{cases}
$$

where $p^{m}$ is the total number of symbols in a sequence of length $K$ having $d(k)=m$. As $K$ is sufficiently large, the sample mean can approximate to zero even when $p^{m}$ is odd. Consequently,

$$
\frac{1}{K} \sum_{k=0}^{K-1} \gamma_{N+k+q}=\frac{1}{K} \sum_{m} \sum_{k=0}^{K-1} \alpha_{N+k+q}^{m} \beta_{N+k+q}^{m} \approx 0
$$

Exploiting above fact, (12) can be simplified to

$$
\bar{x}(t)=p_{0}\left(t-t_{\phi}\right)+p_{0}\left(t+T_{s}-t_{\phi}\right), \quad t \in\left[0, T_{s}\right)
$$

From (16), it is clear that $\bar{x}(t)$ will have non-zero region only around $t_{\phi}$. Exploiting the zeros guards, the objective function to estimate $t_{\phi}$ can be developed as

$$
\hat{t}_{\phi}=\arg \max _{t_{\epsilon} \in\left[0, T_{s}\right)} J\left(t_{\epsilon}\right), J\left(t_{\epsilon}\right)=\int_{0}^{T_{f}}\left[\bar{x}\left(t+t_{\epsilon}\right) \bmod T_{s}\right]^{2} d t
$$

where $\left(\bmod T_{s}\right)$ is included as $\bar{x}(t)$ has size $T_{s}$ while integration in (17) needs periodic extension of $\bar{x}(t)$. In the sequel, we will show that $J\left(t_{\epsilon}\right)$ achieves its unique maximum only at $t_{\phi}$ i.e. $J\left(t_{\phi}\right)=\int_{0}^{T_{f}} p_{0}^{2}(t) d t$. Let $\Delta t=t_{\epsilon}-t_{\phi}$ be the relative misalignment between $t_{\phi}$ and candidate time shift $t_{\epsilon}$ with $\Delta t \in\left(-T_{s}, T_{s}\right)$. As value of $\Delta t$ leads to different results, we consider the two cases: $\Delta t \in\left(-T_{s}, 0\right]$ and $\Delta t \in\left(0, T_{s}\right]$, separately.

Specifically, if $\Delta t \in\left(-T_{s}, 0\right]$, the objective function $J\left(t_{\epsilon}\right)$ can be given as

$$
J\left(t_{\epsilon}\right)=\int_{0}^{T_{f}} p_{0}^{2}(t+\Delta t) d t+\int_{0}^{T_{f}} p_{0}^{2}\left(t+T_{s}+\Delta t\right) d t
$$

Recalling that $p(t)$ has a finite non-zero support within $\left[0, T_{p}\right)$, we get $J\left(t_{\epsilon}\right)=\int_{0}^{T_{f}+\Delta t} p_{0}^{2}(t) d t+\int_{T_{s}+\Delta t}^{T_{f}} p_{0}^{2}(t) d t$, which can be rearranged as

$$
J\left(t_{\epsilon}\right)=J\left(t_{\phi}\right)-\int_{T_{f}+\Delta t}^{T_{s}+\Delta t} p_{0}^{2}(t) d t
$$

Clearly, the objective function $J\left(t_{\epsilon}\right)$ is lower bounded by positive integral $\int_{T_{f}+\Delta t}^{T_{s}+\Delta t} p_{0}^{2} d t>0$, thus yielding a unique maximum if and only if (iff) $\Delta t=0$ or $t_{\epsilon}=t_{\phi}$. Likewise, following the same steps when $\Delta t \in\left[0, T_{s}\right)$, we obtain

$$
J\left(t_{\epsilon}\right)=J\left(t_{\phi}\right)-\int_{T_{f}+\Delta t-T_{s}}^{\Delta t} p_{0}^{2} d t
$$

Again, by a similar argument, we can conclude that $J\left(t_{\epsilon}\right)$ will achieve its maximum iff $t_{\epsilon}=t_{\phi}$, thus validating the algorithm.

Due to complexity restraints, it is sometimes desirable to evaluate $J\left(t_{\epsilon}\right)$ over a finite grid of values $t_{\epsilon}=k T_{\text {sam }}$ rather than a continuous search over $\left[0, T_{s}\right)$, at the expense of reduced synchronization accuracy. It is worth mentioning that our algorithm can estimate $t_{\phi}$ with any possible resolution $T_{\text {sam }}$ and only limited by the affordable complexity of system. Focusing on frame level synchronization with $T_{\text {sam }}=T_{f}$ which is considered to be reasonable compromise between complexity and accuracy, $t_{\phi}$ can be approximated by $t_{\phi}=\hat{k} T_{f}$ where $\hat{k}$ can be obtained by the following discrete optimization

$$
\hat{k}=\arg \max _{k \in\left[0, N_{f}\right)} J(k), J(k)=\int_{0}^{T_{f}}\left[\bar{x}\left(t+k T_{f}\right) \bmod T_{s}\right]^{2} d t
$$

Expressing $t_{\phi}$ in an integral multiple of $T_{f}$ and a residue, i.e. $t_{\phi}=k_{\phi} T_{f}+\delta$ where $N_{\phi}=\left\lfloor t_{\phi} / T_{f}\right\rfloor(\lfloor$.$\rfloor is the integer$ floor operation) and following (18)-(20), the objective function in $(21)$ in IFI/ISI free scenario can be shown as

$$
J(k)= \begin{cases}J\left(t_{\phi}\right)-\int_{T_{f}-\delta}^{T_{f}} p_{0}^{2}(t) d t, & k=k_{\phi} \\ J\left(t_{\phi}\right)-\int_{0}^{T_{f}-\delta} p_{0}^{2}(t) d t, & k=\left[k_{\phi}+1\right]_{\bmod N_{f}} \\ 0 & \text { otherwise }\end{cases}
$$

where the first term in the right hand side represents energy of truncated head $p_{0}(t-\delta)$ while the second one shows energy of truncated tail $p_{0}\left(t+T_{f}-\delta\right)$ of $p_{0}(t)$. The maximum of 
$J(k)$ will be one of these terms, thus affectively estimating the boundary between two consecutive symbols with an ambiguity less than $T_{f}$, which is basically the aim of frame level synchronization.

\section{Demodulation}

The detection statistic for $i^{t h}$ symbol in conventional correlation based Rake receiver is given as

$$
\hat{d}(i)=\arg \max _{m \in[0, M)}\left|\int_{0}^{T_{s}} x\left(t+i T_{s}+\hat{t}_{\phi}\right) v_{m}(t) d t\right|^{2}
$$

where

$$
v_{m}(t)=\sum_{l=0}^{L-1} \hat{\lambda}_{l} g_{T, m}\left(t-\hat{\tau}_{l, 0}\right)
$$

is the reference signal with $\left\{\hat{\lambda}_{l}, \hat{\tau}_{l, 0}\right\}_{l=0}^{L-1}$ representing estimated channel parameters and $g_{T, m}(t)$ is as given in (2). It is worth mentioning at this point that the reservation of one frame for synchronization purpose does not influence the BER performance to large extent. For example, considering binary modulation and correlation based detection as in (23), the BER of modified signalling in (3) can be found as

$$
\begin{aligned}
P_{e} & =\frac{1}{4} \operatorname{erfc}\left(\sqrt{\frac{E_{b}}{2 N_{0}}}\right)+\frac{1}{4} \operatorname{erfc}\left(\frac{N_{f}-2}{N_{f}} \sqrt{\frac{E_{b}}{2 N_{0}}}\right) \\
& \approx \frac{1}{2} \operatorname{erfc}\left(\sqrt{\frac{E_{b}}{2 N_{0}}}\right)
\end{aligned}
$$

where $\operatorname{erfc}(x)=(2 / \sqrt{\pi}) \int_{x}^{\infty} e^{-t^{2}} d t$ is the complementary error function and the approximation is due to fact that $N_{f}$ is chosen sufficiently large in IR-UWB in order to increase effective SNR per symbol, thus resulting in $\left(N_{f}-2\right) / N_{f} \approx 1$. This BER expression in (24) is essentially same as that of conventional orthogonal signalling of (2) found in [7]. Also this information-free frame is only employed over $K$ symbols during synchronization phase, which is less than $1 \%$ of the total transmission block. Thus it is reasonable to conclude the BER performance degradation to be insignificant with our proposed signal format.

\section{Simulations AND COMPARISONS}

In this section, simulations are carried out to evaluate the performance of proposed synchronization algorithm in terms of normalized mean square error (NMSE), probability of acquisition $\left(P_{A}\right)$ and BER against $\mathrm{E}_{\mathrm{x}} / \mathrm{N}_{0}$ (where $\mathrm{E}_{\mathrm{x}}$ is energy within one pulse). In all ensuing simulations, we have used specially designed orthogonal UWB pulses $\left\{\psi_{i}(t)\right\}_{i=0}^{M-1}$ with duration $T_{\psi}=1.28 \mathrm{~ns}$ formed by a linear combination of $\mathrm{B}$ splines along with genetic algorithm based optimization [13]. These pulses satisfy simultaneously the requisites of orthogonality, compatibility with FCC mask and spectral efficiency (which is up to $90 \%$ in our case). Each symbol consists of $N_{f}=10$ frames with $T_{f}=12.8 \mathrm{~ns}$, resulting in symbol duration $T_{s}=128 \mathrm{~ns}$. We have deliberately employed no TH codes $\left\{c_{j}\right\}_{j=0}^{N_{f}-1}=0$ as we are focusing on single user case here.The multipath channel employed in simulations is CM1 indoor channel proposed by IEEE 802.15.3a working group [14]. The synchronization parameter $t_{\phi}$ is randomly generated from a uniform distribution over $\left[0, T_{s}\right)$ at each Monte Carlo trial. We have focused only on frame-level coarse synchronization to keep the simulation time within acceptable limit.

Firstly, NMSE (MSE normalized w.r.t $T_{s}^{2}$ ) performance is evaluated with $K=16,64$ in IFI/ISI free case and the resulting curves are plotted in Fig. 2. The channel response is truncated to $11.52 \mathrm{~ns}\left(T_{\psi}+\tau_{L-1,0} \leq T_{f}\right)$ and then energy normalized to unity, in order to achieve IFI/ISI free condition. It is evident that all NMSE curves decrease monotonically

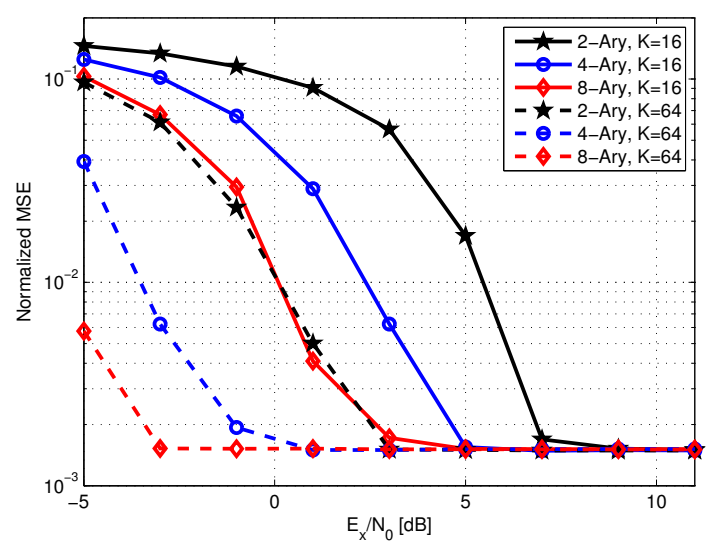

Figure 2. Normalized MSE performance in the absence of IFI/ISI

before flattening out at high SNR. This flattening is induced due to coarse synchronization with limited $T_{f}$ resolution and tend to diminish with increased resolution. Also, it is visible that algorithm remains equally valid with all modulation indices, even with as few as $K=16$ symbols, thus enabling faster synchronization.

Next, the accuracy of proposed algorithm is assessed in terms of acquisition probability. The acquisition probability

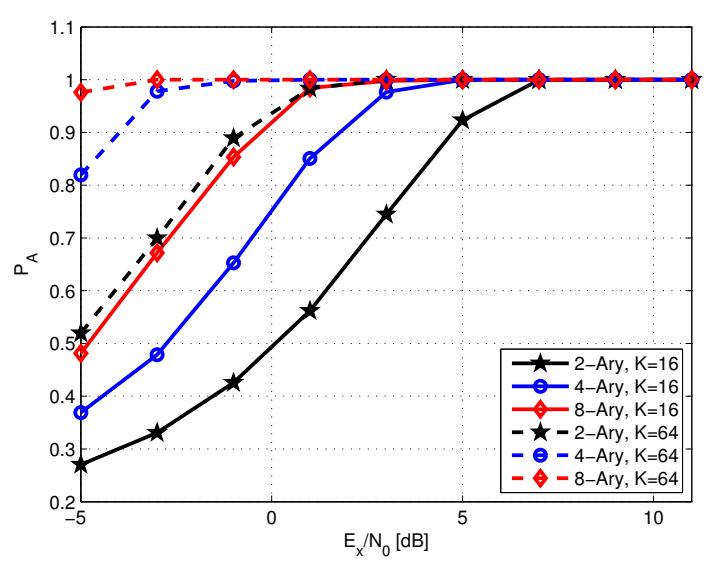

Figure 3. Probability of acquisition performance in the absence of IFI/ISI 
is defined as the probability that $\left|\hat{t}_{\phi}-t_{\phi}\right| \leq T_{f}$. The result in Fig. 3 is consistent with NMSE performance in Fig. 2, showing almost perfect coarse synchronization even at $\mathrm{E}_{\mathrm{x}} / \mathrm{N}_{0}=-5 \mathrm{~dB}$ for 8 -ary modulation with $K=64$ symbols.

Now, we incorporate the effect of IFI/ISI and see the impact on the performance of proposed algorithm. The IFI/ISI is introduced by increasing channel delay spread up to $37.12 \mathrm{~ns}\left(3 T_{f}-T_{\psi}\right)$, thereby inducing an effective two frame IFI. Also by this setting, each symbol is disturbing the first two frames of subsequent symbol, resulting in small ISI. The results are shown in Fig. 4. The performance is almost identical

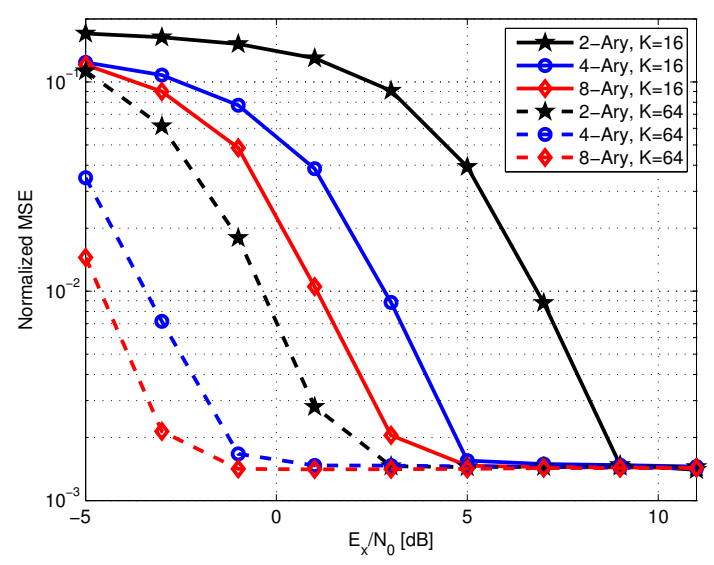

Figure 4. Normalized MSE performance in the presence of IFI/ISI

to Fig. 2 with slight degradation, thus proving the robustness against IFI and a small ISI. This result is logical as the chosen settings will result in non-zero terms for $k=\left[k_{\phi}+n\right] \bmod N_{f}$ with $n \in(0,3)$ in (22). However, the diminishing power profile of CM1 will ensure that the maximum again exists for either $n=0$ or $n=1$, thus retaining the validity of algorithm.

Finally, we test symbol-error-rate (SER) performance with proposed synchronization and Fig. 5 depicts the resulting curves. We perform fine synchronization using (17) and set $N_{f}=3$ to limit the simulation time. To calculate SER, we first estimate $\hat{t}_{\phi}$ with $K=32$ and then demodulate $10^{3}$ symbols using Rake as in (23) at each trial, repeating this over $10^{4}$ trials and then averaging them. We also assume that the channel estimation is done after synchronization and is perfect, resulting in ideal reference signal and thus leaving synchronization error as the only performance limiting factor. It is evident that SER performance with proposed synchronization method is very close to perfect timing even with $K=32$ symbols for all modulation indices, with a slight difference at very low SNR.

\section{CONCLUSIONS AND PERSPECTIVES}

In this paper, we examined one of the most critical issue in IR-UWB i.e. synchronization and developed a blind synchronization algorithm for orthogonal PSM modulation. Exploiting a judiciously designed signal format enables faster synchronization using simple overlap-add operation followed

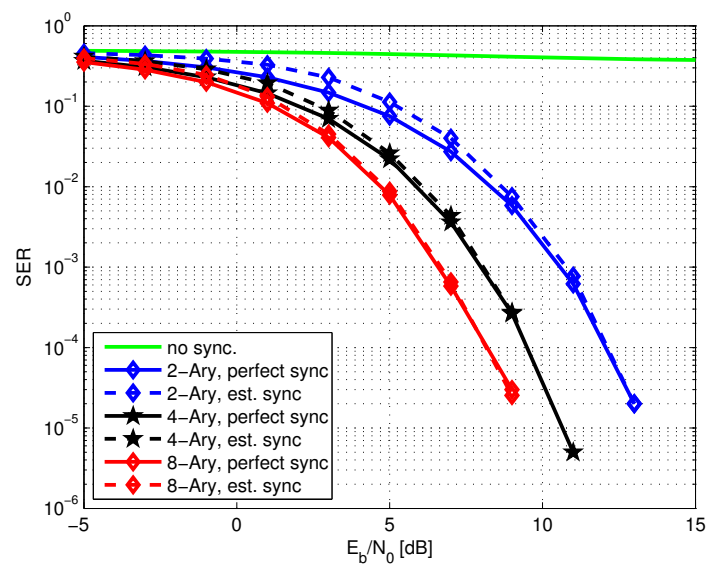

Figure 5. SER performance with perfect and estimated synchronization using $K=32$ symbols in the absence of IFI/ISI

by energy detection. Simulation results are provided to validate the proposed method and its robustness in the presence of IFI and a small ISI. In future, we will focus on extending this method to multi-user scenarios and also will address its analytical performance.

\section{REFERENCES}

[1] L. Yang and G. B. Giannakis, "Ultra-wideband communications: An idea whose time has come," IEEE Sig. Process. Mag., vol. 21, no. 6, pp. 26-54, Nov. 2004.

[2] Z. Tian and G. B. Giannakis, "BER sensitivity to mistiming in ultrawideband impulse radios- part I: Nonrandom channels," IEEE Trans. on Sig. Process., vol. 53, no. 4, pp. 1550-1560, April 2005.

[3] N. He and C. Tepedelenlioglu, "Performance analysis of non-coherent UWB receivers at different synchronization levels," IEEE Trans. on Wireless Commun., vol. 5, no. 6, pp. 1266-1273, June 2006.

[4] Y. Ying, M. Ghogho, and A. Swami, "Code-assisted synchronization for UWB-IR systems: Algorithms and analysis," IEEE Trans. on Sig. Process., vol. 56, no. 10, pp. 5169-5180, Oct. 2008.

[5] G. T. F. de Abreu, C. J. Mitchell, and R. Kohno, "On the design of orthogonal pulse-shape modulation for UWB systems using Hermite pulses," J. of Commun. and Networks., vol. 5, no. 4, p. 328-343, Dec. 2003, special issue on UWB Communications.

[6] M. Ghavami, L. B. Michael, S. Haruyama, and R. Kohno, "A novel UWB pulse shape modulation system," Kluwer Intl. J. on Wireless Pers. Commun., vol. 23, no. 1, pp. 105-120, Aug. 2002.

[7] J. G. Proakis, Digital communications, 4th ed. McGraw-Hill, 2000.

[8] L. Yang and G. B. Giannakis, "Timing ultra-wideband signals with dirty templates," IEEE Trans. on Commun., vol. 53, no. 11, pp. 1952-1963, Nov. 2005.

[9] M. Ouertani, H. Xu, H. Besbes, L. Yang, and A. Bouallègue, "Orthogonal bi-pulse UWB: Timing and (de)modulation," ELSEVIER Physical Communication, vol. 1, no. 4, pp. 237-247, Dec. 2008.

[10] X. Luo and G. B. Giannakis, "Low-complexity blind synchronization and demodulation for (ultra-)wideband multi-user ad hoc access," IEEE Trans. on Wireless Commun., vol. 5, no. 7, pp. 1930 -1941, July 2006.

[11] Y. Qiao, T. Lv, and L. Zhang, "A new blind synchronization algorithm for UWB-IR systems," in proc. IEEE VTC'09, Spain, April 2009, pp. $1-5$.

[12] B. Liu, T. Lv, and H. Gao, "Blind synchronization and demodulation for noncoherent ultra-wideband system with robustness against ISI and IFI," in proc. IEEE ICC'10, South Africa, May 2010, pp. 1-5.

[13] M. Matsuo, M. Kamada, and H. Habuchi, "Design of UWB pulses based on B-splines," in proc. IEEE Intl. Symp. on Circuits and Systems, Japan, May 2005, pp. vol. 6, pp. 5425-5428.

[14] J. R. Foerster, M. Pendergrass, and A. F. Molisch, "A channel model for ultra wideband indoor communication," in proc. Intl. Symp. on Wireless Pers. Multimedia Commun., Japan, Oct. 2003. 